\title{
Constructing of Highly Ordered 3D Network of Carbon Nanotube inside Polymer Matrix and the Improvements in Properties of the Composites
}

\author{
Liang Yang ${ }^{\dagger}$, Yan Zheng ${ }^{\dagger}$, Min Hou, Wanyi Chen, Zhaoqun Wang* \\ Department of Polymer Science and Engineering, School of Chemistry and Chemical Engineering, Nanjing University, Nanjing, P. R. China \\ Email address: \\ zqwang@nju.edu.cn (Zhaoqun Wang) \\ ${ }^{*}$ Corresponding author \\ $\dagger$ Liang Yang and Yan Zheng are co-first authors. \\ To cite this article: \\ Liang Yang, Yan Zheng, Min Hou, Wanyi Chen, Zhaoqun Wang. Constructing of Highly Ordered 3D Network of Carbon Nanotube inside \\ Polymer Matrix and the Improvements in Properties of the Composites. American Journal of Polymer Science and Technology. \\ Vol. 5, No. 1, 2019, pp. 9-15. doi: 10.11648/j.ajpst.20190501.12
}

Received: January 21, 2019; Accepted: February 28, 2019; Published: March 21, 2019

\begin{abstract}
In the past few decades, carbon nanotube-filled polymer composites have attracted the attention of many researchers with their excellent performance. However, the currently known methods of preparing composite materials do not maximize the performance of the carbon nanotubes themselves. In this work, by using our proposed "particle-constructing" method, multi-wall carbon nanotubes (MWCNTs) connected with each other to form highly ordered 3D network structure in polystyrene (PS) matrix. The strategy contains two steps as follows. First, MWCNTs-coated PS composite particles were prepared by the thermodynamic driving heterocoargulation method, without any requirement to surface modification or surface treatment whether for the MWCNTs or the PS microspheres. Then, the resultant MWCNTs-coated PS composite particles are used as building blocks to fabricate the highly ordered 3D MWCNT-based PS composite materials by a general compression mould at room temperature and a subsequent heat treatment at an appropriate temperature. We discuss in detail the effects of PS particle size, oxidation of MWCNTs and their length on the electrical conductivity of materials. The fabricated MWCNTbased PS composite materials exhibited excellent properties such as a much higher electrical and mechanical properties. Moreover, the method and process are pretty simple, convenient and environment-friendly for obtaining the unique composite structure and excellent properties.
\end{abstract}

Keywords: Carbon Nanotube, Conductive Material, Polymer Nanocomposites, 3D Network Structure

\section{Introduction}

Carbon nanotubes possess high mechanical strength, electrical and thermal conductivity, and unique optical and electronic properties. These properties make carbon nanotubes valuable for a wide range of applications. [1-11] In recent years, considerable efforts have been made to fabricate different carbon morphologies and explore their application in various fields, including composites, electrochemical devices, field emission devices, nanoscale electronic devices, and sensors. In order to fully utilize the superior performance of Multi-wall carbon nanotubes (MWCNTs), a feasible approach is to prepare MWCNT-based polymer composites. Naturally, the key to the preparation of MWCNT-based composites with excellent properties is the uniform distribution of MWCNTs in the polymer matrix. To maximize the advantage of MWCNTs as effective reinforcement for high performance polymer composites, the MWCNTs should not form aggregates, must be well dispersed to enhance the interfacial interaction with the matrix. Several processing methods available for fabricating MWCNT-based polymer composites based on either thermoplastic or thermosetting matrices have been studied, including solution mixing, $[12,13]$ in situ polymerization, $[14,15]$ melt blending [16-21] and so on. Ajayan [12] et al. dispersed MWCNTs in ethanol by sonication and mixed them 
with a mixture of epoxy monomer and curing agent under mechanical stirring. After evaporation of the volatile solvent, the MWCNT-epoxy mixture was poured into capsular molds and then cured. Electron microscopy images of sample slices showed a partial alignment of the MWCNTs on the cut surface. Loos [22] et al. first dispersed MWCNTs in solvent with sodium dodecyl sulfate, then mixed the dispersions with polystyren (PS) latex, froze the mixture in liquid nitrogen and freeze-dried it overnight to obtain composite power. However, all of the solutions mentioned above have disadvantages of complicated processing, high energy consumption and unfriendly environment. In addition, the uniformity of the MWCNTs dispersed in the matrix cannot be effectively controlled by these methods and that's not to mention establishing a highly ordered 3D network structure in the composites.

Herein, a simple and efficient method is developed for preparing MWCNT-based polymer composites. Through this method, MWCNTs can form a complete and densitycontrolled 3D ordered network structure in the matrix. In this strategy, MWCNTs-coated polymer microspheres are prepared by thermodynamic driving heterocoagulaition method and then, the resulting composite particles are subjected to molding and subsequent heat treatment to obtain composites, inside which the MWCNTs on surface of the composite particles connect each other to form a complete network. Therefore, it can be called as "particle-constructing" method, which is quite distinct from the widely used conventional random liquid-phase blend. In this article, the effects of matrix particle size, oxidization and length of carbon nanotube on the electrical conductivity of the composites were investigated systematically.

\section{Experimental Section}

\subsection{Materials}

Styrene (AR) obtained from Shanghai Chemical Reagent Co., was purified by column chromatography. Azodiisobutyronitrile (AIBN) of chemical reagent grade (Shanghai Chemical Reagent Co.) was purified by recrystallization in 95\% ethanol. Polyvinylpyrrolidone (PVP) with an average molar mass of $58 \mathrm{~kg} / \mathrm{mol}$ (PVP K-30) was purchased from Acros Organics. Absolute ethanol, $95 \%$ ethanol, nitric acid and isopropanol were purchased from Nanjing Chemical Reagent Co. and used as received. Short multi-wall carbon nanotubes (diameter: 20-30 nm, length: 0.5-2 $\mu \mathrm{m}$ ) were purchased from Chengdu Organic Chemicals Co., Ltd. and long Multi-wall carbon nanotubes (diameter: 20-40 nm, length: $\geq 5 \mu \mathrm{m}$ ) were purchased from Shenzhen Nanotech Port Co., Ltd.

\subsection{Synthesis of Polystyrene (PS) Microspheres}

PS microspheres were prepared by dispersion polymerization. To get PS microspheres with average diameter of 2-3 $\mu \mathrm{m}$, PVP stabilizer $(2.5 \mathrm{~g})$, AIBN (0.2 g) and styrene monomer $(20 \mathrm{~g})$ were dissolved in isopropanol (160 $\mathrm{mL}$ ) in a four-necked round-bottom flask purged with nitrogen. Then the solution was heated to $70^{\circ} \mathrm{C}$ and maintained for $24 \mathrm{~h}$ with vigorous mechanical stirring before cooling down to ambient temperature. Subsequently, the PS microspheres were transferred from isopropanol to deionized water by repeated centrifugation and redispersion process, and finally diluted with deionized water to $10 \mathrm{wt} \%$ solid content before use.

\subsection{Fabrication of Oxidized MWCNTs (O-MWCNTs)}

First, $1 \mathrm{~g}$ of MWCNT was placed in a $150 \mathrm{ml}$ three-necked flask, and then $100 \mathrm{~mL}$ of concentrated nitric acid was added. After 1 hour of ultrasonication, the system was reacted at $80^{\circ} \mathrm{C}$ for 24 hours. The finally obtained product was washed with a large amount of deionized water and dried in an oven.

\subsection{Preparation of PS/MWCNTs Composite Particles}

Briefly, MWCNTs $(20 \mathrm{mg})$ were added into ethyl alcohol $(20 \mathrm{~mL})$ under mechanical stirring, companied with ultrasonic treatment for $1.5 \mathrm{~h}$. Add PS emulsion $(10 \mathrm{~g}, 10$ $\mathrm{wt} \%$ ) into the flask and the mixture was stirred for another $0.5 \mathrm{~h}$. Then remove water from the suspension by centrifugation. Finally, the composite particles were dried at $60^{\circ} \mathrm{C}$ in vacuum drying oven. The preparation process of $\mathrm{PS} / \mathrm{O}-\mathrm{MWCNTs}$ nanocomposite particles is similar to the above.

\subsection{Fabrication of the MWCNTS-based PS Composite Materials}

The PS/MWCNTs composite particles powders were pressed into wafer in a powder tableting machine with 800 $\mathrm{MPa}$, and the samples were then heated at $130^{\circ} \mathrm{C}$ for $1 \mathrm{~h}$.

\subsection{Characterization}

Scanning electron microscope (SEM) images were taken on an S-4800 instrument (Hitachi Co., Japan). Raman spectra were collected on an Aramis confocal microscope Raman spectrometer (Horiba Johib Yvon, Edison, NJ). A 633 nm HeNe laser was used as the excitation light source and kept below $0.5 \mathrm{~mW}$ to prevent thermal damage of the samples. The spatial resolution of the beam spot was around $1 \mu \mathrm{m}^{2}$, attained using a $100 \times$ objective microscope lenses. The electric conductivity of the composites was tested by a four probe tester (ST2263, Suzhou Jingge Electronic Co., Ltd.) and the mechanical properties of the material are tested by dynamic thermomechanical analysis $(\mathrm{DMA}+450$, France 01dB-Metravib) using a double cantilever mode with a heating rate of $2^{\circ} \mathrm{C} \mathrm{min}^{-1}$ and $1 \mathrm{~Hz}$. 


\section{Results and Discussion}

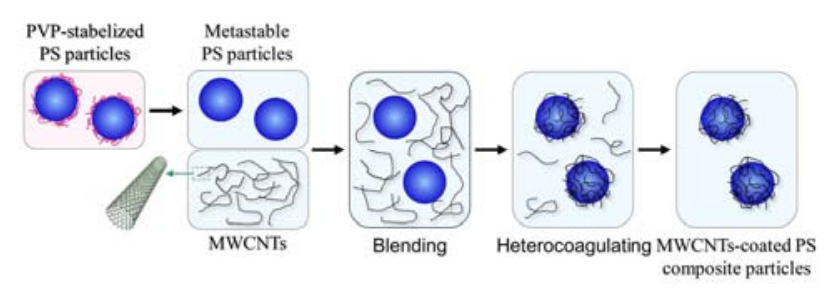

Figure 1. Schematic diagram of the preparation process of MWCNT-coated PS composite particles by using thermodynamic driving heterocoagulation method.

In this system, monodisperse PS microspheres prepared by conventional dispersion polymerization are used without any surface modification and functionalization. As shown in Figure 1, the PS microspheres thus got no chemically bound ionic groups and then were shifted into a metastable state through sufficient purification to remove most stabilizer polyvinylpyrrolidone (PVP). By a simple blending of PS and MWCNTs, the MWCNTs were willing to attach upon the metastable PS microspheres. It is thought that the lowest Gibbs free energy is reached when unstable or metastable colloidal particles are covered by the MWCNTs. In this approach, the thermodynamic effect is utilized as a unique driving force for the heterocoagulation between the metastable PS microspheres and the MWCNTs. It is particularly noteworthy that the MWCNTs were used as original form without any surface modifications or surface treatments and thus, both the MWCNTs and PS microspheres are unstable in water. However, the MWCNTs coating onto the PS microspheres can reduce the total interface area of the PS microspheres and MWCNTs to water and then reduce the total interfacial energy of the system. This thermodynamic driving effect has been demonstrated in many binary colloidal mixtures using different nano-sized functional units. [23] Furthermore, as the MWCNTs clung to the surface of PS microspheres at a fairly close distance, strong $\pi$ electrons interaction should begin to take effect, which is derived from aromatic organic molecules and the basal plane of carbon nanotubes.

Figure 2 shows scanning electron microscopy (SEM) images of PS/MWCNTs composite particles with different weight ratios of MWCNTs. Compared with the smooth surface of original PS microspheres, many carbon nanotubes can be obviously observed on the particle surface and the nanotubes overlap with each other. At higher weight ratio, the large numbers of MWCNTs randomly and uniformly covered on the PS microspheres that look like a dense network. When the ratio reaches about $7 \mathrm{wt} \%$, the PS microsphere is almost fully wrapped by the nanotubes.

The difference in MWCNTs coverage degree was further demonstrated by the Raman spectra as shown in Figure 3. For the PS microspheres (curve a), very strong signal peaks appeared at about 625 and $1000 \mathrm{~cm}^{-1}$, but the G peak and D peak of MWCNTs at about 1600 and $1330 \mathrm{~cm}^{-1}$ are displayed obviously in curves b-e for the PS/MWCNTs composite particles prepared using different MWCNTs content. More importantly, as the mass fraction of MWCNTs in the composite microspheres increases, the characteristic signal peaks of PS gradually weaken. This phenomenon is considered to be a powerful proof for the gradient changes in coverage degree of MWCNTs since the Raman signal belonging to PS core could be obscured or absorbed by the MWCNTs layer.
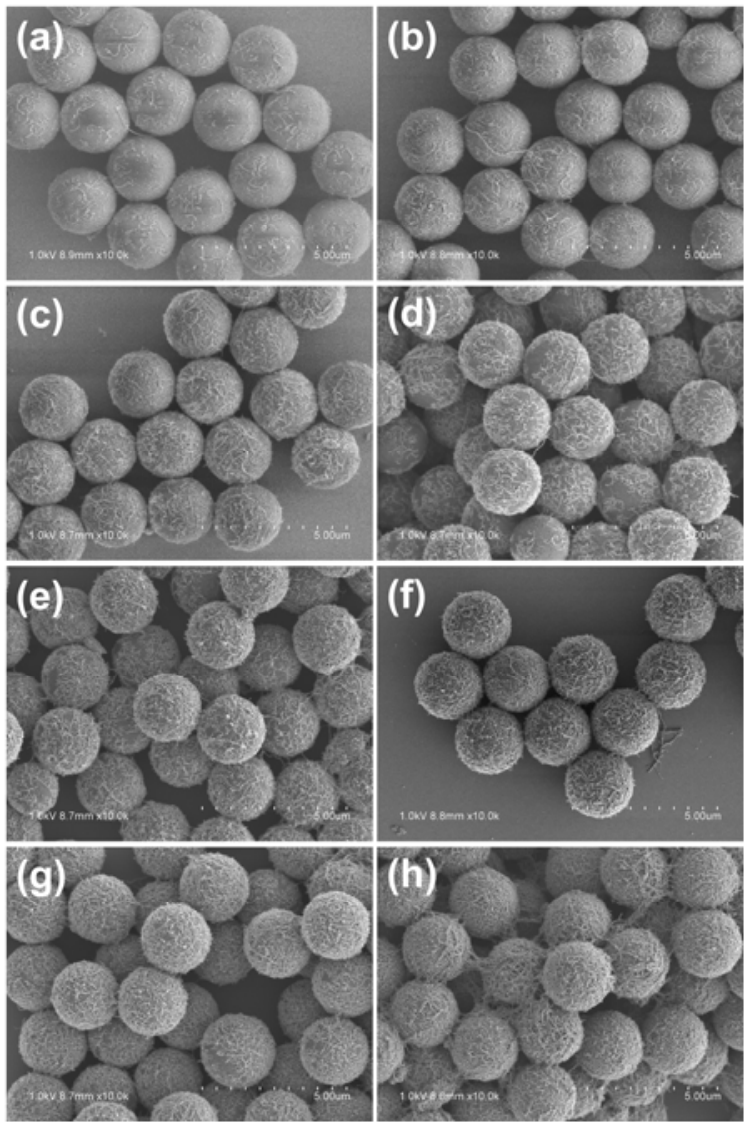

Figure 2. SEM images of PS/MWCNTs composite particles prepared with different MWCNTs/PS weight ratio of 1 wt\% (a), 2 wt \% (b), $3 w t \%$ (c), 4 $w t \%(d), 5 w t \%(e), 6 w t \%(f), 7 w t \%(g)$ and $10 w t \%(h)$, respectively.

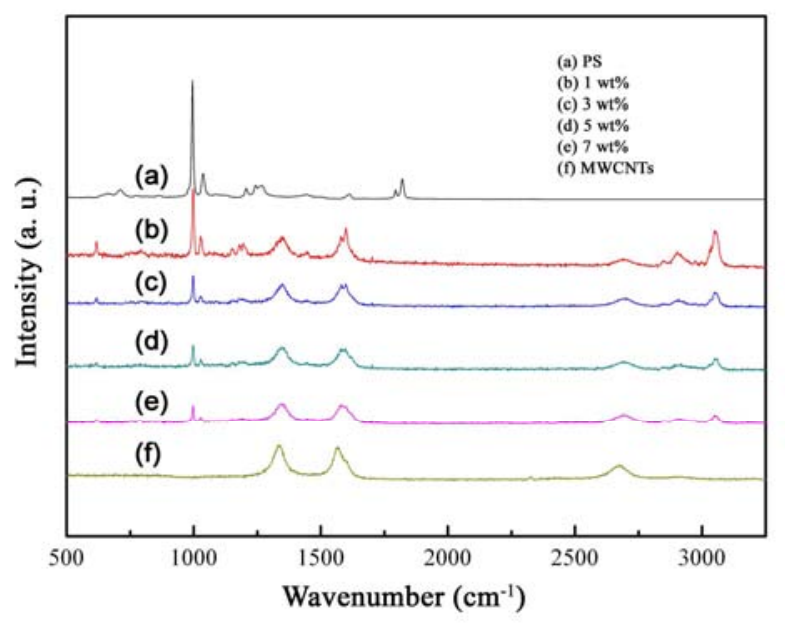

Figure 3. Raman spectra of PS microspheres (a), PS/MWCNTs composite particles prepared with different $M W C N T_{s} / P S$ weight ratio of 1 wt\% (b), 3 $w t \%(c), 5 w t \%(d), 7 w t \%(e)$ and MWCNTs (f). 
It is important to note that a very simple method was adopted to successfully obtain the PS/MWCNTs composite particles and particularly, the surface morphology and structure of the composite particles were effectively controlled. In the process, any surface functionalization or surface modification were entirely unnecessary whether for the PS microspheres or the carbon nanotubes, which is obviously a breakthrough in preparing the analogous composite particles. The dramatic phenomenon and interesting results benefit from the proposed thermodynamic driving heterocoargulation method. As mentioned above, the bare PS particles without PVP are in a metastable state in water, so the MWCNTs tend to attach on the PS particles to lower the total Gibbs free energy of the colloid system on the basis of thermodynamic viewpoint. Therefore, according to the theory, the MWCNTs coverage of the composite particles can be governed by some other manners besides the weight ratio of PS/MWCNTs, for example, addition of fourth component into the colloid systems. As indicated by SEM images in Figure 4, when adding different amount of PVP into the repeatedly washed PS latex before blending it with the MWCNTs, gradually lowered MWCNTs coverage was got, even down to almost zero as adding PVP with $1.0 \mathrm{wt} \%$ of the PS. Just as we expected, as more and more PVP molecules were added into the system, the PS microspheres and MWCNTs can respectively stabilize in water since the PVP should readily attach to their surface. As a result, the MWCNTs coverage can be powerfully controlled by changing the amount of post-added PVP.
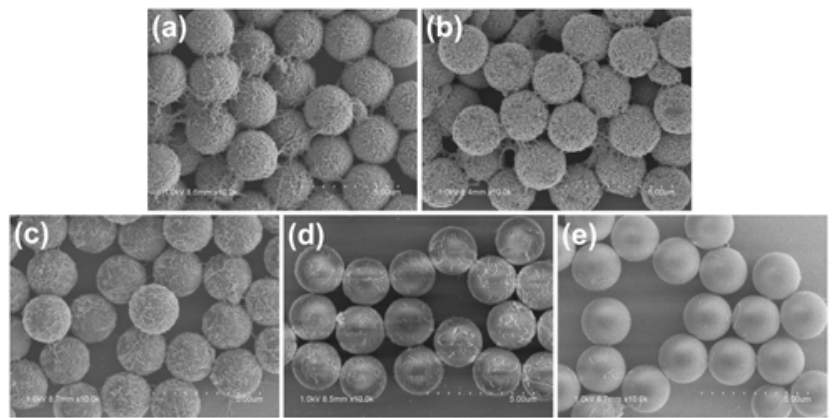

Figure 4. PS/MWCNTS composite particles prepared by pre-mixing the PS microspheres with PVP at different PVP/PS weight ratio of 0 wt $\%$ (a), 0.25 $w t \%(b), 0.50 w t \%(c), 0.75 w t \%(d)$ and $1.0 w t \%(e)$, respectively, when the $M W C N T s / P S$ weight ratio remained constant at $10 \mathrm{wt} \%$.

Using the resulting PS/MWCNTs composite particles as building units, we can construct MWCNTs-based composites with highly ordered 3D composite structure, for which there are only simple two steps as illustrated in Figure 5. Figure 5b shows cross sectional image of the MWCNTs-based PS composites prepared by molding the PS/MWCNTs composite particles (as shown in Figure 5a) under $800 \mathrm{MPa}$ at room temperature. The individually arrayed PS/MWCNTs composite particles can be still observed but they are deformed into general hexagonal blocks as expected. Figure $5 \mathrm{c}$ shows the morphology of the same sample after heating at $130^{\circ} \mathrm{C}$ for $1 \mathrm{~h}$. Significant change is observed in the cross- sectional structure, in which the interface among the particles disappears and the MWCNTs connect with each other to form a complete network. That is, a very perfect, regular and well-defined 3D MWCNTs network was successfully woven throughout the composite material via the simple and common molding process. In a real sense, it is the strict cross section of MWCNTs and PS region, whereas the samples without the heat treatment were broken almost at the interface among the original composite particles. At a larger magnification (Figure 5d), we can get a better view of the section morphology of fractured MWCNTs. Based on these phenomena, it is certain that the PS chains and MWCNTs are closely combined with each other. In brief, to the best of our knowledge, such a highly ordered 3D MWCNTs-based composite material embedded with an intact, uniform and well-defined MWCNTs network had not been reported before. Moreover, almost no toxic organic solvent was used throughout the preparation process so that this method can be said to be an environmentally friendly technology.
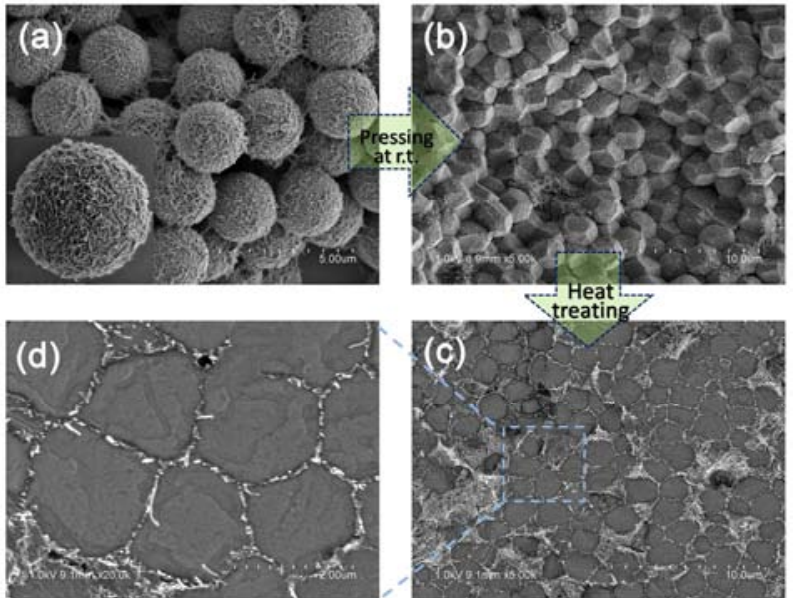

Figure 5. SEM images of PS/MWCNTs composite particles (a), cross-section of MWCNTs-based PS composite material obtained by mold pressing the PS/MWCNTs composite particles at 800 MPs (b), subsequently heat-treating at $130^{\circ} \mathrm{C}$ for $1 \mathrm{~h}(\mathrm{c})$ and a high magnification of a local image (d).
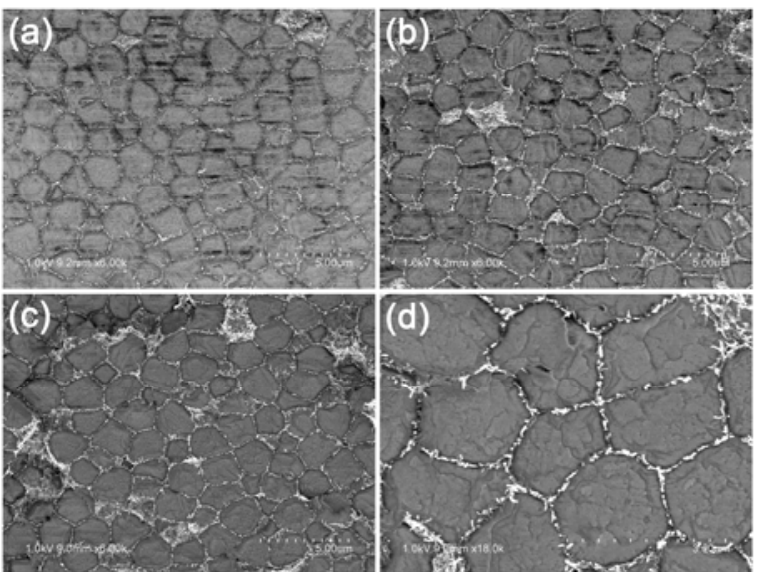

Figure 6. Cross-sectional SEM images of the highly ordered $3 D$ PS/MWCNTS composite materials prepared by the proposed particleconstructing method with different weight loadings of MWCNTs: $3 \mathrm{wt} \%$ (a), $5 w t \%(b), 7 w t \%(c, d)$. 
Figure 6 shows cross sectional images of a series of MWCNTs-based PS composite materials that were fabricated using the PS/MWCNTs composite particles with different coverage of MWCNTs. The intact and clear network structure can be observed on the cross section of the composites as the PS microspheres had a bushy overlayer of MWCNTs. However, as using the composite particles with a lower MWCNT coverage (Figure 2c), we can still obtain an intact MWCNT network inside the pressing block as shown in Figure 6a. Obviously, the carbon nanotubes from the

(a)

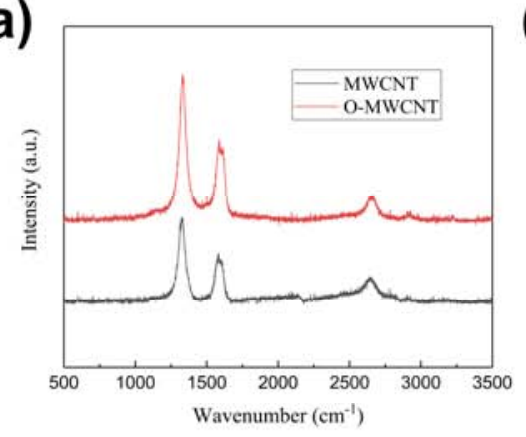

(d)

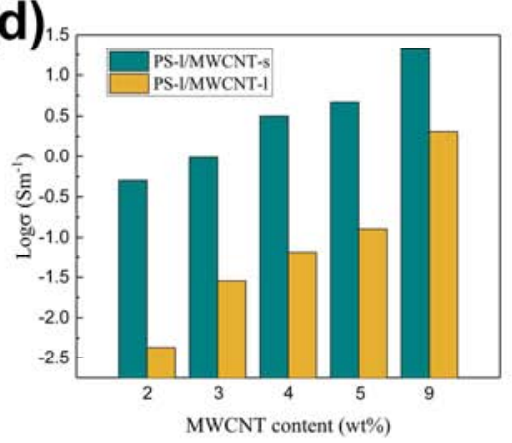

(b)

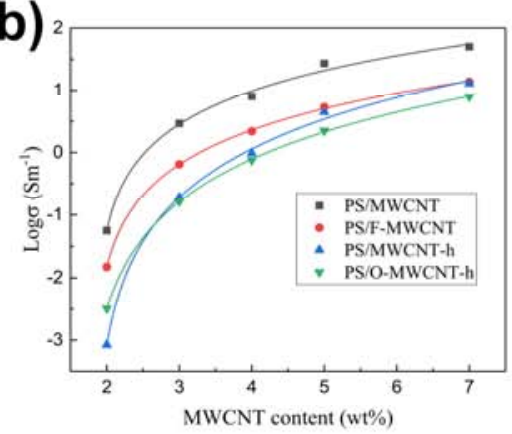

(e)

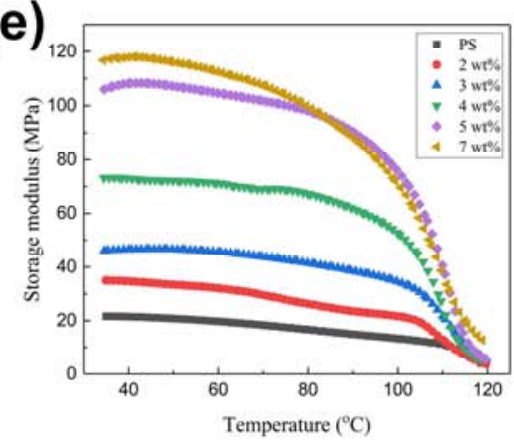

different composite particles could overlap and interweave with each other to form a continuous route way though the PS microspheres covered with relatively sparse MWCNTs. In fact, the MWCNTs-based PS composite materials had a bicontinuous structure after heat-treating the pressing blocks at $130^{\circ} \mathrm{C}$. The interparticle diffusion of the PS chains through the space between the nanotubes should result in the disappearance of particle interface to form the PS continuous phase in the composite materials.

Figure 7. The Raman spectrum of carbon nanotubes before and after oxidization that are marked as MWCNTs and O-MWCNTs (a); the conductivity diagram of the composites prepared by using MWCNTs and O-MWCNTs before and after heat treatment (b), the composites prepared by using MWCNTs and PS particles with different sizes of 2.5 and $4.5 \mu \mathrm{m}$, marked as PS-s and PS-l (c) and the composites prepared by using PS-l and MWCNTs with different lengths marked as MWCNT-l and MWCNT-s (d); the storage modulus of the composites prepared by using different MWCNT content at different temperature (e) and storage modulus of composites at $35^{\circ} \mathrm{C}(f)$.

The highly ordered 3D carbon nanotube network and PS phase bicontinuous structure undoubtedly can make a great contribution to the properties of the composite materials, especially to the electrical and mechanical properties. Figure 7 shows the effects of oxidation and length of the carbon nanotubes, size of the PS particles on the electrical conductivity and the mechanical properties of the composites. Obviously, the Raman spectrum of Figure 7a shows a certain increase in $\mathrm{I}_{\mathrm{D}} / \mathrm{I}_{\mathrm{G}}$ after acidification of MWCNTs (labeled as O-MWCNTs) due to the introduction of more defects by oxidation. The conductivity of the composites prepared by MWCNTs that were not oxidized is obviously higher than that of the composites prepared by the O-MWCNTs. This performance improvement obviously benefits from the integrity of hyperconjugation structure of unoxidized MWCNTs, fully revealing importance of the thermodynamic driving heterocoargulation method, by which the carbon nanotubes are not necessary to oxidize for preparing PS/MWCNTs composite particles. After heat treatment of the

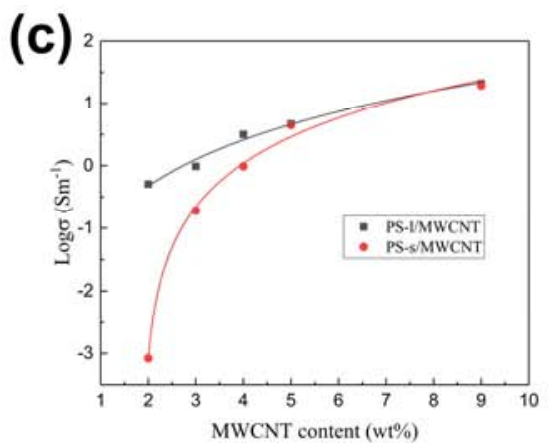

(f)

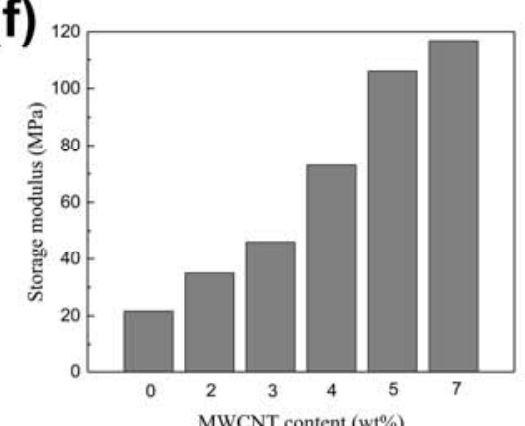

cold-pressed composites, the electrical conductivity was decreased to some extent whether the carbon nanotubes were oxidized or not oxidized, as show as Figure $7 \mathrm{~b}$. The decline in conductivity should be blamed on diffusion of PS chains to the intersections between the overlapping carbon nanotubes to segregate them. The effect of PS particle size on the electrical conductivity of the composite is shown in Figure 7c. It is clear that the composite prepared by the larger PS particles (PS-1) has a higher conductivity. This is due to the larger the PS particle size, the smaller the total surface area, and the larger PS particles can be covered with more MWCNTs at the same filler content, as shown in Figure S1. But, along with increase of the MWCNT content, the difference in conductivity caused by the particle size becomes smaller. Particularly, they have a nearly same conductivity and it rose slowly with the MWCNT content after about $7 \mathrm{wt} \%$, which suggests that both PS particles can be completely covered with MWCNTs at this content and a good conductive path formed in matrix. In addition, the 
percolation thresholds of PS-s/MWCNT and PS-1/MWCNT are 1.07 and 0.282 vol\% respectively (see Figure S2). It exhibited a decrease as increasing PS particle size because a lower MWCNT content is required to form the percolative pathways as using the large PS particles that have small specific surface. These results indicated that increasing the relative size of PS particle to carbon nanotube should be favorable to improvement of the electrical properties in general. As shown in Figure 7d, the length of the carbon nanotubes is also an important factor on the electrical conductivity of the composites. The PS/MWCNT-s composites prepared from short MWCNTs had a higher conductivity than the long MWCNTs (i.e. PS/MWCNT-1 composites). In fact, it has the same reason with the size of the PS particles, that is, the relative size of PS particle to carbon nanotube essentially influences the conductivity of composites. But, besides the essential, there is one more that is conformation of the tubes. The long MWCNTs are easy to form entanglement and accumulation that should cause their inhomogeneous distribution on the PS microspheres, especially at low MWCNT contents. In general, the PS/MWCNT composite materials exhibited excellent electrical properties due to the 3D MWCNT network structure. The conductivity reported in this work is several orders of magnitude higher than the classical solution mixing and melt mixing methods reported in the literature. [21, 24, 25] In order to better prove this point, PS/MWCNT composites were prepared using MWCNTs and O-MWCNTs (taking $7 \mathrm{wt} \%$ of MWCNT content for instance) by solution mixing method. As shown in Figure S3, it is apparent that the composite prepared by the particle construction method has a conductivity that is four orders of magnitude higher than the contrast samples.

Similar to the electrical properties, increasing the MWCNTs loading can significantly increase the storage modulus of the PS/MWCNTs composites, as shown in Figure 7e and 7f. When the MWCNT content was 2, 3, 4, 5 and 7 $\mathrm{wt} \%$, the storage modulus of the PS/MWCNTs composites increased by $62.0 \%, 113.0 \%, 238.1 \%, 390.2 \%$ and $440.0 \%$ to homogeneous PS material, respectively. The excellent mechanical properties of the composites demonstrate the unique advantages of this bicontinuous structure.

\section{Conclusion}

The MWCNT-based PS composite materials were fabricated by using the proposed "particle-constructing" method, which was divided into two steps: firstly, preparing the MWCNTs-coated PS composite particles by the thermodynamic driving heterocoargulation method and then, pressing the resulting PS/MWCNTs composite particles to fabricate the highly ordered 3D MWCNT-based PS composite materials at room temperature with a subsequent heat treatment at an appropriate temperature. The significant results can be summarized as follows: (a) the composites embedded with an intact, uniform and well-defined MWCNTs 3D network, forming the PS phase and MWCNT network bicontinuous structure; (b) the preparation method and process are pretty simple and convenient, without any requirement to surface modification or surface functionalization whether for the carbon nanotubes or the PS microspheres, and they are also environment-friendly due to almost no requirement to organic solvents during the whole process; (c) the composite materials exhibited excellent electrical properties with a substantial improvement compared to the solution mixing method, and particularly, there was a further increase in conductivity due to using the unoxidized MWCNTs; and (d) the storage modulus of the PS/MWCNTs composites significantly increased than the homogeneous PS material, obviously owing to the uniform and regular dispersion of MWCNTs and the bicontinuous structure. The thermodynamic driving heterocoargulation method and "particle-constructing" technique for fabricating the unique composite materials has high controllability over composite structure and should be universal for other MWCNTs-based composites.

\section{Supporting Information}

The following files are available free of charge.

The SEM image of PS-1/MWCNTS, the conductivity fitting of the composite and the conductivity comparison of the composites prepared by different methods. (PDF)

\section{Author Contributions}

The manuscript was written through contributions of all authors. All authors have given approval to the final version of the manuscript. $\$$ These authors contributed equally.

\section{Notes}

The authors declare no competing financial interest.

\section{Acknowledgements}

This work was supported by Program for Changjiang Scholars and Innovative Research Team in University (IRT1252) and the Fundamental Research Funds for the Central Universities.

\section{References}

[1] Z. Spitalsky, D. Tasis, K. Papagelis, C. Galiotis, Carbon nanotube-polymer composites: Chemistry, processing, mechanical and electrical properties, Progress in Polymer Science, 35 (2010) 357-401.

[2] D. M. Guldi, G. M. A. Rahman, F. Zerbetto, M. Prato, Carbon nanotubes in electron donor-acceptor nanocomposites, Accounts of Chemical Research, 38 (2005) 871-878.

[3] D. Tasis, N. Tagmatarchis, A. Bianco, M. Prato, Chemistry of carbon nanotubes, Chemical Reviews, 106 (2006) 11051136. 
[4] P. R. Bandaru, Electrical properties and applications of carbon nanotube structures, Journal of Nanoscience and Nanotechnology, 7 (2007) 1239-1267.

[5] J. P. Salvetat, J. M. Bonard, N. H. Thomson, A. J. Kulik, L. Forro, W. Benoit, L. Zuppiroli, Mechanical properties of carbon nanotubes, Applied Physics a-Materials Science \& Processing, 69 (1999) 255-260.

[6] E. W. Wong, P. E. Sheehan, C. M. Lieber, Nanobeam mechanics: Elasticity, strength, and toughness of nanorods and nanotubes, Science, 277 (1997) 1971-1975.

[7] M. M. J. Treacy, T. W. Ebbesen, J. M. Gibson, Exceptionally high Young's modulus observed for individual carbon nanotubes, Nature, 381 (1996) 678-680.

[8] S. J. Tans, M. H. Devoret, H. J. Dai, A. Thess, R. E. Smalley, L. J. Geerligs, C. Dekker, Individual single-wall carbon nanotubes as quantum wires, Nature, 386 (1997) 474-477.

[9] M. R. Maschmann, G. J. Ehlert, B. T. Dickinson, D. M. Phillips, C. W. Ray, G. W. Reich, J. W. Baur, Bioinspired Carbon Nanotube Fuzzy Fiber Hair Sensor for Air-Flow Detection, Advanced Materials, 26 (2014) 3230-+.

[10] C. Mu, Y. Song, W. Huang, A. Ran, R. Sun, W. Xie, H. Zhang, Flexible Normal-Tangential Force Sensor with Opposite Resistance Responding for Highly Sensitive Artificial Skin, Advanced Functional Materials, 28 (2018).

[11] L.-C. Jia, Y.-K. Li, D.-X. Yan, Flexible and efficient electromagnetic interference shielding materials from ground tire rubber, Carbon, 121 (2017) 267-273.

[12] P. M. Ajayan, O. Stephan, C. Colliex, D. Trauth, ALIGNED CARBON NANOTUBE ARRAYS FORMED BY CUTTING A POLYMER RESIN-NANOTUBE COMPOSITE, Science, 265 (1994) 1212-1214.

[13] F. M. Du, J. E. Fischer, K. I. Winey, Coagulation method for preparing single-walled carbon nanotube/poly (methyl methacrylate) composites and their modulus, electrical conductivity, and thermal stability, Journal of Polymer Science Part B-Polymer Physics, 41 (2003) 3333-3338.

[14] M. Wang, K. Zhang, X.-X. Dai, Y. Li, J. Guo, H. Liu, G.-H. Li, Y.-J. Tan, J.-B. Zeng, Z. Guo, Enhanced electrical conductivity and piezoresistive sensing in multi-wall carbon nanotubes/polydimethylsiloxane nanocomposites via the construction of a self-segregated structure, Nanoscale, 9 (2017) $11017-11026$

[15] S. Kumar, T. D. Dang, F. E. Arnold, A. R. Bhattacharyya, B. G. Min, X. F. Zhang, R. A. Vaia, C. Park, W. W. Adams, R. H. Hauge, R. E. Smalley, S. Ramesh, P. A. Willis, Synthesis, structure, and properties of $\mathrm{PBO} / \mathrm{SWNT}$ composites, Macromolecules, 35 (2002) 9039-9043.

[16] H. J. Barraza, F. Pompeo, E. A. O'Rear, D. E. Resasco, SWNT-filled thermoplastic and elastomeric composites prepared by miniemulsion polymerization, Nano Letters, 2 (2002) 797-802.

[17] R. Haggenmueller, H. H. Gommans, A. G. Rinzler, J. E. Fischer, K. I. Winey, Aligned single-wall carbon nanotubes in composites by melt processing methods, Chemical Physics Letters, 330 (2000) 219-225.

[18] M. A. Lopez-Manchado, L. Valentini, J. Biagiotti, J. M. Kenny, Thermal and mechanical properties of single-walled carbon nano tubes-polypropylene composites prepared by melt processing, Carbon, 43 (2005) 1499-1505.

[19] P. Potschke, A. R. Bhattacharyya, A. Janke, Carbon nanotubefilled polycarbonate composites produced by melt mixing and their use in blends with polyethylene, Carbon, 42 (2004) 965969.

[20] K. Wu, C. Lei, R. Huang, W. Yang, S. Chai, C. Geng, F. Chen, Q. Feng, Design and Preparation of a Unique Segregated Double Network with Excellent Thermal Conductive Property, Acs Applied Materials \& Interfaces, 9 (2017) 7637-7647.

[21] S. Biswas, I. Arief, S. S. Panja, S. Bose, AbsorptionDominated Electromagnetic Wave Suppressor Derived from Ferrite-Doped Cross-Linked Graphene Framework and Conducting Carbon, Acs Applied Materials \& Interfaces, 9 (2017) 3030-3039.

[22] J. Yu, K. Lu, E. Sourty, N. Grossiord, C. E. Konine, J. Loos, Characterization of conductive multiwall carbon nanotube/polystyrene composites prepared by latex technology, Carbon, 45 (2007) 2897-2903.

[23] Y. Li, Z. Wang, C. Wang, Y. Pan, H. Gu, G. Xue, Colloid thermodynamic effect as the universal driving force for fabricating various functional composite particles, Langmuir: the ACS journal of surfaces and colloids, 28 (2012) 1270412710 .

[24] J. Hwang, J. Jang, K. Hong, K. N. Kim, J. H. Han, K. Shin, C. E. Park, Poly (3-hexylthiophene) wrapped carbon nanotube/poly (dimethylsiloxane) composites for use in finger-sensing piezoresistive pressure sensors, Carbon, 49 (2011) 106-110.

[25] T. Li, L.-F. Ma, R.-Y. Bao, G.-Q. Qi, W. Yang, B.-H. Xie, M.-B. Yang, A new approach to construct segregated structures in thermoplastic polyolefin elastomers towards improved conductive and mechanical properties, Journal of Materials Chemistry A, 3 (2015) 5482-5490. 